\title{
Rapid prototyping IoT solutions based on Machine Learning
}

\author{
Antonio Rizzo \\ Università di Siena \\ via Roma 56 - Siena \\ Italy \\ antonio.rizzo@unisi.it \\ Antonio Gisondi \\ Università di Siena \\ via Roma 56 - Siena \\ Italy \\ antonio.gisondi@udoo.org
}

\author{
Francesco Montefoschi \\ Università di Siena \\ via Roma 56 - Siena \\ Italy \\ francesco.montefoschi@unisi.it \\ Giovanni Burresi \\ Università di Siena \\ via Roma 56 - Siena \\ Italy \\ giovanni.burresi@udoo.org
}

\author{
Maurizio Caporali \\ Università di Siena \\ via Roma 56 - Siena \\ Italy \\ maurizio.caporali@unisi.it \\ Roberto Giorgi \\ Università di Siena \\ via Roma 56 - Siena \\ Italy \\ giorgi@dii.unisi.it
}

\begin{abstract}
Nowadays Machine Learning (ML) has reached an all-time high, and this is evident by considering the increasing number of successful start-ups, applications and services in this domain. ML techniques are being developed and applied to an ever-growing range of fields, from on-demand delivery to smart home. Nevertheless, these solutions are failing at getting mainstream adoption among interaction designers due to high complexity. In this paper we present the integration of two Machine Learning algorithms into UAPPI, our open source extension of the prototyping environment MIT App Inventor. In UAPPI much of the complexity related to ML has been abstracted away, providing easy-to-use graphical blocks for rapid prototyping Internet of Things solutions. We report on limits and opportunities emerged from the first two scenario-based explorations of our design process.
\end{abstract}

\section{CCS CONCEPTS}

- Human-centered computing $\rightarrow$ Human computer interaction (HCI) - Computing methodologies $\rightarrow$ Machine learning • Computer systems organization $\rightarrow$ Embedded and cyberphysical systems

\section{KEYWORDS}

Permission to make digital or hard copies of all or part of this work for personal or classroomuse is granted without fee provided that copies are not made or distributed for profit or commercial advantage and that copies bear this notice and the full citation on the first page. Copyrights for components of this work owned by others than ACM must be honored. Abstracting with credit is permitted. To copy otherwise, or republish, to post on servers or to redistribute to lists, requires prior specific permission and/or a fee. Request permissions from permissions@acm.org ECCE 2017, September 19-22, 2017, Umeå, Sweden

(C) 2017 Association for Computing Machinery.

ACM ISBN 978-1-4503-5256-7/17/09...\$15.00 DOI: $10.1145 / 3121283.3121291$
App Inventor, UDOO, Machine Learning, Rapid Prototyping, Support Vector Machine, Google Vision

\section{AC M Reference format:}

A. Rizzo, F. Montefoschi, M. Caporali, A. Gisondi, G. Burresi, and R. Giorgi. 2017. Rapid prototyping IoT solutions based on Machine Learning. In Proceedings of European Conference on Cognitive Ergonomics 2017 (ECCE 2017), Umeå, Sweden, September 19-22, 2017, 4 pages.

https://doi.org/10.1145/3121283.3121291

\section{INTRODUCTION}

Machine Learning is a research field at the intersection of statistics, artificial intelligence, and cognitive science. Nowadays ML has reached an all-time high, and this is evident by considering the increasing number of successful start-ups, applications and services in this domain [1]. From automatic recommendations of movies to watch, what food to order or which products to buy, to personalized online radio and recognizing your friends in your photos, many modern services and devices have ML algorithms at their core. When we use websites like Facebook, Amazon, or Netflix, it is very likely that part of the site uses ML models. It is about extracting knowledge from data to improve the user exp erience.

Although humans are an integral part of the learning process, traditional ML systems used in these applications are agnostic to the fact that inputs/outputs are from/for humans. Furthermore, even though human application of ML algorithms to real-world problems requires embedding the algorithms in systems of some sort, and the form and usability of these systems impact the feasibility and efficacy of applied ML work, research at the intersection of HCI and ML is still rare.

Yet, the interest at this intersection of ML with HumanComputer Interaction (HCI) is growing. It aims at making interaction with humans a central part of developing ML systems. These efforts include applying interaction design principles to ML 
systems, using human-subject testing to evaluate ML systems and inspire new methods, and changing the input and output channels of ML systems to better leverage human capabilities [2].

In the following, we introduce a rapid prototyping environment we are developing that integrates $\mathrm{ML}$ libraries into our open source extension of App Inventor: UAPPI (UDOO App Inventor) [3]. Such integration allows to rapidly create prototypes of applications for cyber-physical systems, able to identify and classify scenarios according to values measured by sensors.

We present our first version of the tool though examples of use applied to smart home. To this aim, we first shortly introduce the UDOO X86 prototyping board, the UAPPI IDE with ML components, and we finally illustrate its use through the smart home scenarios.

\section{UDOO X86}

UDOO X86 (Fig. 1) is a prototyping single board computer based on Intel processors. Like the other boards of the UDOO family, it embeds on the same Printed Circuits Board (PCB) a high performance multicore processor and an Arduino compatible microcontroller unit.

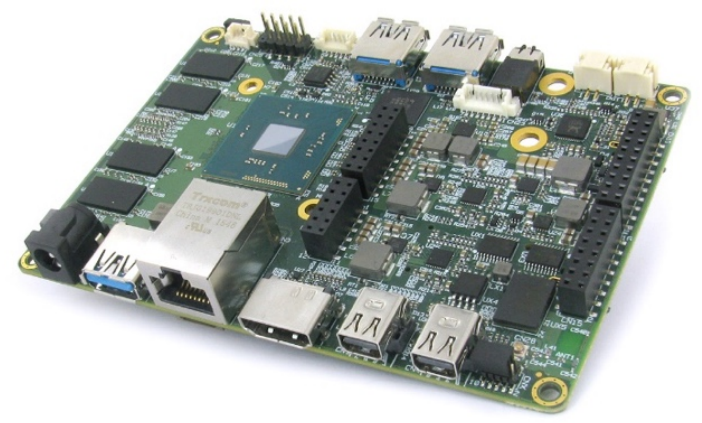

Figure 1: The UDOO X86 single board computer.

The board was designed to provide high computational power and high graphics performance. Being fully compatible with the X86 architecture, it allows users to install the most common operating systems in their official and original versions. The UDOO X86 board integrates a full Arduino 101, based on the Intel Curie chip. The standard Arduino pinout allows programmers to directly use all the official Arduino tools and libraries, plug shields and connect sensors. The integration on the same PCB of a fully-fledged computer and one of the most versatile microcontrollers easily allows rapid prototyping of IoT solutions [4].

\section{UDOO APP INVENTOR}

App Inventor for Android is a visual programming platform for creating mobile apps for Android based smartphones and tablets. Originally developed by Google, it is now maintained by the MIT [5]. Developing applications in App Inventor does not require writing classic source code. The look and behavior of the app is developed visually, using a series of building blocks for each intended graphical or logical component. App Inventor aims to make programming enjoyable and accessible to novices, makers and designers.

Our extension of App Inventor, UAPPI, provides a seamless developing environment between Arduino and Android. Makers, designers, and students have a tool that can be used to develop applications by providing must-have functionalities like GUIs, network access and storage on databases, and by incorporating the popular Arduino sensors and actuators. In short, UAPPI integrates two worlds, the computer (Android) and the microcontroller (Arduino) in a seamless environment where it is possible to envision and prototype interactive solutions for the digital world and the IoT [6].

\section{INTERACTIVE MACHINE LEARNING}

$M L$ is transforming the way we interact with products and with the whole ecosystem of technologies that go es under the umbrella of Internet of Things. So far, most of these changes occur bey ond any awareness and conscious involvement of the final users. ML algorithms are designed to evolve and change based on data that's either fed into a system or collected in real-time. Algorithms processes the data, draws conclusions and makes inferences to provide users content that's tailored specifically for them. However standard ML algorithms are non-interactive: they input training data and output a model.

Interactive Machine Learning (IML) refers to an approach for letting users interactively train representation parameters with rapid, focused, and incremental model updates. The model construction process typically involves a training loop wherein the user repeatedly chooses, is given or produce example data, and then provides a judgment or label with which to train the system. As it is an interactive process, these systems are collectively known IML [7]. Implementing ML solutions is hard for novices. It requires a solid math and computer science background. To get satisfactory results, ML algorithms need to be tuned and optimization parameters regulated. But since the dawn of the new millennium there is evidence showing that users - even users who are not ML experts - can often construct good classifiers [8]. But without playing with $\mathrm{ML}$ algorithms in a tangible and real context it is hard to understand its fundamental basis, and to get them working properly, even at prototype-level accuracy. For this aim, we have introduced a new category of blocks in UAPPI, named "Machine Learning", which provides easy to use components. Those components can be seen as black boxes, that, for what is possible, will automatically tune. This first release includes two well established components, a generic data classifier and a face detector. We will introduce them contextually to an applicative example.

\section{SMART HOME SCENARIOS}


We are developing our ML extension for UAPPI by prototyping appliances for the smart home following a scenario-based approach. Smart homes, and similar IoT contexts, require more and more designing for embedded interactions. These interactions are embedded within our environment, they put more emphasis on the context created rather than on a specific device, and sometime their design may even eschew the conventions of HCI. Instead of focusing on creating dedicated GUIs, these interactions are rooted in the space and in the objects that populate it. Embedded interactions try to exploit the existing physical resources for producing emerging behaviors in the interactions rather than overlapping graphic interfaces to generate such behaviors. IML offers the opportunity to exploit sensors and actuators available in the IoT contexts for generating such embedded interactions. Here we present two simple scenarios, they involve the same entities but connected with different relations. The entities are: the environment, the users, and the ML model. The environment is the physical space where users perform and receive actions. ML Model represents an autonomous agent that can be trained to classify data, take decisions and perform actions. The user is our main actor, the person we are designing for.

The two scenarios differ either for the kind of sensors involved and for the use of the ML algorithm. The first scenario is based on gas sensors for collecting data from the environment and use a generic data classifier that needs to be trained to proper classify the data. The second scenario uses an off-the-shelf USB webcam to get some facial features using a pre-trained model.

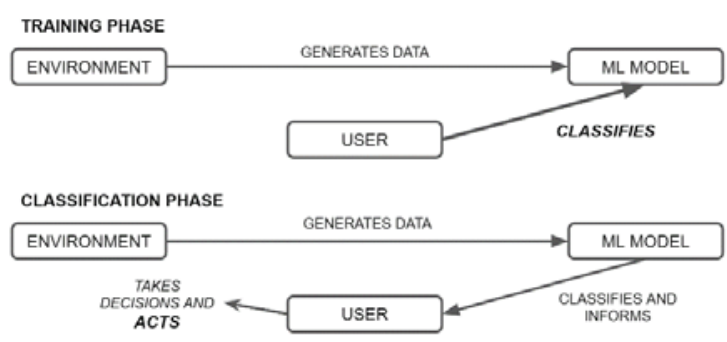

Figure 2: First scenario, training and classifications phases.

In the first scenario, the environment generates data collected by sensors. This data is used to generate the ML Model with the contribution of the user in the training process (see Fig. 2). The user labels the samples based on her personal needs or wishes, directly interacting with the Model in a personalized perspective. In the classification phase the ML Model classifies the data basing on criteria built on the user "wishes". The ML Model informs the user about what is happening and helps him to take decision or actions in the environment (e.g. eating the overripe banana or trashing the rotten ones).

In the second scenario, the user does not need to contribute to the generation of the ML Model, which is pre-trained. Furthermore, it is the user who generates the data that are classified by the Model and program the actions the Model can take according to the results of the classification [9] (see Fig. 3).
The different relations among the entities requires different tools and facilities in the prototyping tool, and this is just what we put under exploration in this early phase of our design process. The analyses were carried out on a UDOO X86, Android-x86 7.1.1 and the latest version of UAPPI [10].

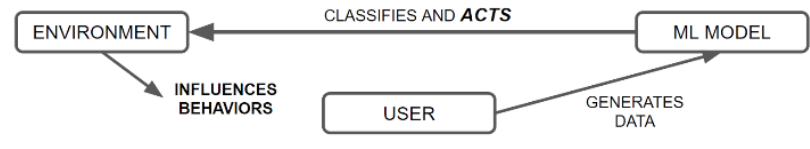

Figure 3: Second S cenario, classification phase.

\subsection{Environmental data classification with SVM}

The first algorithm considered for prototyping interactions is Support Vector Machine (SVM) [11], a generic data classifier. The SVM is trained by showing it some data samples. Each data sample is composed of a features vector and a label. When some examples are provided, the machine computes a mapping function able to classify unlabeled feature vectors, never seen during the training phase. UAPPI uses libsvm [12] to provide UdooSvm, a component which allow both the training and the classification phases.

Feature vectors can be generated by other blocks, specific to the required task, like analyzing an image, or reading data from some Arduino digital and/or analog pins. In this scenario, we want to get the ripeness level of some bananas, distinguishing between 3 categories: ripe fruit, overripe fruit and rotten fruit. We expected the bacteria to release gases during their activity on the fruit. We connected an array of different Arduino gas sensors to the analog input pins to measure different gases, since we did not know the kind and level of gases a priori.

We left two bananas for 9 days inside a wood box with several holes, to let air flow through. On the top of the box we taped the gas sensors array (MQ-x). Data collected during the first 6 days were tagged as "ripe fruit". In the next two days, bananas ripened more and more, and data points were tagged as "overripe". Subsequent data were tagged as "rotten fruit", as banana peels were totally black. After providing the examples, it is possible to build the model. In UAPPI, users can experiment different SVM kernels, using a drop-down menu. Now that we have a model able to classify novel data, adding some logic in UAPPI to announce the fruit state using the text to speech or Twitter is a matter of minutes.

When the relevant features are known, developing a classifier model in UAPPI is not challenging; however, the training phase may be time consuming. A great number of examples should be provided, otherwise the model may not be able to correctly classify novel data, since it will learn just the few examples provided, without gaining the ability to generalize. It follows that developing a custom model is advisable in cases where it is easy to provide sufficient examples for all the classes. Otherwise, it is preferable to use a pre-trained model, as shown in the next section. 


\subsection{Face expression re cognition with Google Vision}

Google Vision [13] is a framework for finding objects in photos and video, using real-time computer vision technology. The mobile face API [14] allows to find human faces in photos, tracks positions of facial landmarks (eyes, nose, and mouth) and provides information about the state of facial features -- Are the subject's eyes open? Are they smiling? UAPPI integrates this library in the UdooVision component. Using a USB camera, it can detect the prominent face in the frame. The component exposes values between 0 and 1 proportionally to how much the eyes are open and how much a person is smiling ( 0 means closed eyes / no smile; 1 means ey es fully opened / full smile). In a few minutes, it is possible to prototype a smart lamp (see Fig. 4).



Figure 4: Facial features extracted by Google Vision.

Winking the left eye, the lamp enters in "programming mode", where it powers up an RGB LED strip proportionally to the smile. Winking again exits the programming mode. In this case it is immediately noticeable that the absence of the training phase simplifies further the use of ML components. The user can focus only on the mapping the classified values into some output or action, rather than on the model building task.

\section{CONCLUSIONS}

Interactive Machine Learning systems enable new ways to design emerging behaviors in the interaction between humans and their environment. IML can be a powerful resource for designing interactions with embedded system in smart environments. These interactions can concern either the use multimodal data as input to produce desiderated outputs, or the generation of new patterns of interactions that exploit the huge amount of data made available by cyber-physical systems. However, to bring ML in a design project, it is mandatory to try to define the learning problem as clearly and fully as possible. What are the input parameters we will provide? What kinds of outputs are we looking for? What kind of training data will exemplify the correlations between these inputs and outputs? If we cannot reason clearly about what a particular component should do and what data it should be trained on, then most likely the ML algorithm won't be able to figure this out either.

The integration of ML algorithms in UAPPI allows users to play with such resources. The data, as in our example, can come from simple Arduino sensors or a webcam. However, at present the facilities so far implemented does not support adequately the situation described in Scenario 1, that is a situation where the designer has to build the model for mapping the inputs to the outputs from scratch. Before to see any preliminary results, it is required the procurement and cleaning of a dataset, the selection of a different $\mathrm{ML}$ algorithm, the exploration of the relevant features and a training process. Instead the same tools so far developed allow to exploit existing ML models and apply them to mapping predefined inputs to desired outputs. UAPPI users are only required to take some creativity and imagine an application that can exploit the potentialities of the features detections implemented in the ready to use model. Making more algorithms/models available in UAPPI will allow users to experiment more and more. And this is the road that we will privilege for the future development of UAPPI.

\section{ACKNOWLEDGMENTS}

We thankfully acknowledge the support of the European Union H2020 program through the AXIOM project (grant ICT01-2014 GA 645496).

\section{REFERENCES}

[1] Michael I Jordan and Tom M Mitchell. 2015. Machine learning: Trends, perspectives, and prospects. Science 349, 6245: 255-260.

[2] Saleema A mershi, Maya Cak mak, William Bradley Knox, and Todd Kulesza. 2014. Power to the people: The role of hu mans in interactive machine learning. AI Magazine 35, 4: 105-120.

[3] Antonio Rizzo, Francesco Montefoschi, Sara Ermini, and Giovanni Burresi. 2015. UDOO App Inventor: Introducing Novices to the Internet of Things. International Journal of People-Oriented Programming (IJPOP) 4, 1: 33-49.

[4] UDOO X86. Retrieved from https://www.udoo.org/udoo-x86/

[5] Hal Abelson. App Inventor for Android. Google Research Blog. Retrieved from https://research.googleblog.com/2009/07/app-inventor-for-android.html

[6] Antonio Rizzo, Giovanni Burresi, Francesco Montefoschi, Maurizio Caporali, and Roberto Giorgi. 2016. Making IoT with UDOO. IxD\&A30: 95-112.

[7] Jerry Alan Fails and Dan R Olsen Jr. 2003. Interactive machine learning. Proceedings of conference on Intelligent user interfaces, ACM, 39-45.

[8] Malcolm Ware, Eibe Frank, Geo ffrey Holmes, Mark Hall, and Ian H Witten. 2001. Interactive machine learning: letting users build classifiers. International Journal of Human-Computer Studies 55, 3: 281-292.

[9] Rebecca Fiebrink, Perry R Cook, and Dan Trueman. 2011. Human model evaluation in interactive supervised learning. Proceedings of the SIGCHI Conference on Human Factors in Computing Systems, ACM, 147-156.

[10] Francesco Montefoschi. UAPPI IDE and Companion source code. Retrieved from https://github.com/fmntf/appinventor-sources

[11] Alex M Andrew. 2000. An introduction to Support Vector Machines and other Kernel-Based learning methods by Nello Christianini and John Shawe-Taylor, Cambridge University Press, Cambridge, 2000, xiii+ 189 pp., ISBN 0-52178019-5 (Hbk, pounds 27.50).-. Robotica 18, 6: 687-689.

[12] Chih-Chung Chang and Chih-Jen Lin. 2011. LIBSVM: a library for support vector machines. ACM transactions on intelligent systems and technology (TIST) 2, 3: 27 .

[13] Mobile Vision. Retrieved from https://developers.google.com/vision/

[14] Google Developers, APIs for Android. Retrieved from https://developers.google.com/android/reference/co m/google/android/gms/visio $\mathrm{n} /$ face/package-summary 